\title{
Measuring multipartite entanglement through dynamic susceptibilities
}

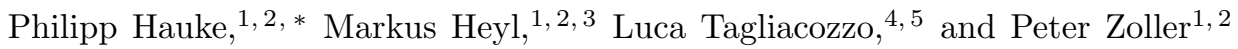 \\ ${ }^{1}$ Institute for Theoretical Physics, University of Innsbruck, 6020 Innsbruck, Austria \\ ${ }^{2}$ Institute for Quantum Optics and Quantum Information of the Austrian Academy of Sciences, 6020 Innsbruck, Austria \\ ${ }^{3}$ Physik Department, Technische Universität München, 85747 Garching, Germany \\ ${ }^{4}$ ICFO-Institut de Ciencies Fotoniques, Mediterranean Technology Park, 08860 Castelldefels (Barcelona), Spain \\ ${ }^{5}$ Department of Physics and Scottish Universities Physics Alliance University of Strathclyde, Glasgow G4 ONG, Scotland, UK
}

Entanglement is considered an essential resource in quantum metrology and quantum computation, and it plays a central role in our understanding of quantum many-body physics. Developing protocols to detect and quantify entanglement of many-particle quantum states is thus a key challenge for present experiments. Here, we show that the quantum Fisher information, a witness for genuinely multipartite entanglement, becomes measurable for thermal ensembles via the dynamic susceptibility, i.e., with resources readily available in present cold atomic gas and condensed-matter experiments. This moreover establishes a connection between multipartite entanglement and many-body correlations contained in response functions, with immediate implications close to quantum phase transitions. There, the quantum Fisher information becomes universal, allowing us to identify strongly entangled phase transitions with a divergent multipartite entanglement. We illustrate our framework using paradigmatic quantum Ising models, and point out potential signatures in optical-lattice experiments and strongly-correlated materials.

While entanglement is central to our understanding of quantum many-body physics 1 , its direct measurement in an actual experiment to characterise quantum phases and phase transitions has remained elusive. In a fewbody quantum system, in particular in a quantum information context involving few qubits, entanglement can be measured and quantified via tomography of the density matrix 2 25. In experiments with few atoms in optical lattices as engineered Bose- or Fermi-Hubbard models, entanglement entropy and purity of quantum states become measurable with protocols based on preparing copies of the quantum system in combination with the unique tools of atomic physics, including single-site manipulation and observation 6, 7. However, an intrinsic feature of these protocols is an exponential scaling of resources, and thus an a priori limit to small system sizes.

In contrast, we will be interested below in quantifying multipartite entanglement for large system size, while still being accessible with experimentally realistic tools and resources. Our approach is based on measurement of the quantum Fisher information (QFI) as a witness of $m$-particle entanglement [8 10, as familiar from quantum metrology [11-13]. As our main result, we will show that the QFI for thermodynamic equilibrium is directly related to measurement of dynamic susceptibilities (Fig. 1). To illustrate detection of entanglement via the QFI, we study several examples of quantum phase transitions (QPTs). We are able to identify a class of strongly entangled QPTs with divergent multipartite entanglement by studying the universal scaling of the QFI [17 $\sqrt{20}$, also at non-zero temperatures (see also Refs. 21 23] for scaling of related quantum metrics). The entanglement quantified through the QFI is not only a resource for quantum metrology [, $[9]$,

\footnotetext{
* philipp.hauke@uibk.ac.at
}
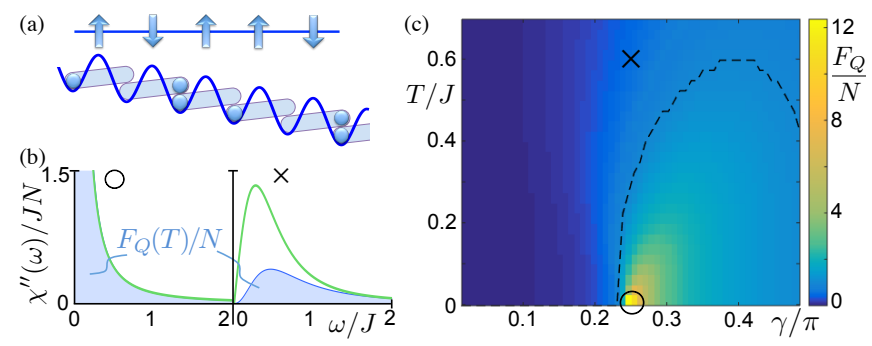

Figure 1. Measurement prescription for the quantum Fisher information (QFI), a witness for multipartite entanglement. (a) A quantum many-body system is prepared in a thermal state at temperature $T$. The example shows at the top an Ising spin chain, Eq. (8), describing solid-state samples [14, 15] and optical-lattice experiments (bottom) [16]. The concept applies also to fermions and bosons, and in any spatial dimension. (b) Standard tools, such as inelastic Bragg or neutron scattering, measure the imaginary part of the dynamic susceptibility $\chi^{\prime \prime}(\omega, T)$ as a function of frequency exchange $\omega$ [green line, computed via Eq. [11]]. The integral $F_{Q}(T)=\frac{4}{\pi} \int_{0}^{\infty} \mathrm{d} \omega \tanh \left(\frac{\omega}{2 T}\right) \chi^{\prime \prime}(\omega, T)$ gives the QFI (shaded areas). (c) This procedure allows mapping out the QFI as a function of temperature and transverse field, parametrized by $\gamma$ (exact data for $N=64$ ). Low-temperature states (circle) can host entanglement, but it is lost at larger temperatures (cross). The quantum Ising chain has divergent entanglement close to the quantum critical point, and a robust entangled region extending to finite temperatures (enclosed by the dashed line).

it also permits the characterisation of exotic quantum effects such as many-body localization 24. While the outstanding feature of the presented protocol is its experimental simplicity, we will also discuss that the QFI as entanglement witness will be blind to non-local entanglement [25, 26], as, e.g., in topological systems.

Background on the quantum Fisher information 
Originally a concept from quantum metrology, the quantum Fisher information, $F_{Q}$, quantifies the maximal precision with which a parameter (a phase) $\vartheta$ can be estimated using a given quantum state $\rho$ [11 13]. For $M$ independent measurements, the variance of $\vartheta$ is bounded by $(\Delta \vartheta)^{2} \geq 1 /\left(M F_{Q}\right)$, the so-called quantum CramérRao bound [11]. A better precision, i.e., a smaller righthand side, can be reached if the state $\rho$ is more sensitive towards a unitary transformation generated by the hermitian operator $\hat{\mathcal{O}}$ associated to $\vartheta$. In other words, the QFI quantifies the distinguishability of $\rho$ from $\rho^{\prime}=\mathrm{e}^{-i \vartheta \hat{\mathcal{O}}} \rho \mathrm{e}^{i \vartheta \hat{\mathcal{O}}}$, for infinitesimal $\vartheta$ (without loss of generality, we set the mean value of $\vartheta$ to 0 ).

For a pure quantum state $\rho=|\psi\rangle\langle\psi|$, such as the ground state of a given Hamiltonian, the QFI assumes the simple form of a connected correlation function, which can be easily computed or measured,

$$
F_{Q}=4 \Delta(\hat{\mathcal{O}})^{2}=4\left(\langle\psi|\hat{\mathcal{O}} \hat{\mathcal{O}}| \psi\rangle-\langle\psi|\hat{\mathcal{O}}| \psi\rangle^{2}\right) .
$$

Matters become much more complicated in a mixed state, such as a thermal ensemble $\rho=\sum_{\lambda} p_{\lambda}|\lambda\rangle\langle\lambda|$, where $|\lambda\rangle$ is the energy eigenbasis with occupation probabilities $p_{\lambda}=\exp \left(-E_{\lambda} / T\right) / Z$, with $Z$ the partition function. In such a case, the QFI takes the considerably more complex structure

$$
F_{Q}=2 \sum_{\lambda, \lambda^{\prime}} \frac{\left(p_{\lambda}-p_{\lambda^{\prime}}\right)^{2}}{p_{\lambda}+p_{\lambda^{\prime}}}\left|\left\langle\lambda|\hat{\mathcal{O}}| \lambda^{\prime}\right\rangle\right|^{2}
$$

(where the sum includes only terms with $p_{\lambda}+p_{\lambda^{\prime}}>0$ ).

Importantly, the quantum Cramér-Rao bound can fall below classical limits if $\rho$ describes an entangled state of $N>1$ particles [13. Consider a local generator $\hat{\mathcal{O}}=$ $\sum_{l=1}^{N} \hat{\mathcal{O}}_{l}$, where $\hat{\mathcal{O}}_{l}$ has a spectrum of unit width (see Methods). If $\rho$ achieves a sufficiently large QFI to break classical bounds,

$$
f_{Q} \equiv F_{Q} / N>m
$$

with $f_{Q}$ the QFI density and $m$ a divisor of $N$, then $\rho$ must be $m+1$-partite entangled [8, 9].

Recently, in a remarkable atomic-gas experiment [10, it has been demonstrated that a lower bound on the QFI can be measured by studying the behaviour of an observable's probability distribution under the unitary transformation $\mathrm{e}^{i \vartheta \hat{\mathcal{O}}}$. This could then be used to demonstrate the presence of bipartite entanglement. Here, we show how the QFI can be measured directly and efficiently for any quantum many-body system in a thermal state at any temperature.

\section{Main result}

As the major result of this work, we rigorously relate the QFI to a Kubo response function,

$$
F_{Q}(T)=\frac{4}{\pi} \int_{0}^{\infty} \mathrm{d} \omega \tanh \left(\frac{\omega}{2 T}\right) \chi^{\prime \prime}(\omega, T),
$$

where $\chi^{\prime \prime}(\omega, T)=\Im(\chi(\omega, T))$ is the imaginary, dissipative part of the dynamic susceptibility in the state $\rho$ with respect to $\hat{\mathcal{O}}$ - the same thermal state and generator for which the QFI is evaluated. A similar connection has already been noted for the related geometric tensor in the ground state [21, 22], and response functions in imaginary time are being used to make the geometric tensor calculable in quantum Monte Carlo computations, see, e.g., Ref. 27. Equation (4) relates the QFI to response functions at non-zero temperatures and in real time, thus providing a tool to measure it directly in laboratory experiments.

Proof: The proof of Eq. (4) is straightforward. It requires only the minimal assumption of thermal equilibrium. Setting $\hbar=1=k_{B}$, the dynamic susceptibility is defined as

$$
\chi(\omega, T)=i \int_{0}^{\infty} \mathrm{d} t \mathrm{e}^{i \omega t} \operatorname{tr}(\rho[\hat{\mathcal{O}}(t), \hat{\mathcal{O}}])
$$

where $\hat{\mathcal{O}}(t)=\mathrm{e}^{i H t} \hat{\mathcal{O}} \mathrm{e}^{-i H t}$. It is convenient to work in the Lehmann representation, i.e., the energy eigenbasis, where

$$
\chi^{\prime \prime}(\omega)=\sum_{\lambda, \lambda^{\prime}}\left(p_{\lambda}-p_{\lambda^{\prime}}\right)\left|\left\langle\lambda|\hat{\mathcal{O}}| \lambda^{\prime}\right\rangle\right|^{2} \pi \delta\left(\omega-E_{\lambda^{\prime}}+E_{\lambda}\right) .
$$

Exploiting that for a thermal state $\int_{-\infty}^{\infty} \mathrm{d} \omega \tanh \left(\frac{\omega}{2 T}\right) \delta\left(\omega-E_{\lambda^{\prime}}+E_{\lambda}\right)=\tanh \left(\frac{E_{\lambda^{\prime}}-E_{\lambda}}{2 T}\right)=$ $\frac{p_{\lambda}-p_{\lambda^{\prime}}}{p_{\lambda}+p_{\lambda^{\prime}}}$, using the asymmetry of $\chi^{\prime \prime}(\omega, T)$, and correcting for prefactors, we directly obtain Eq. (4). This proof can be straightforwardly extended to the QFI matrix, and, via the fluctuation-dissipation theorem to the dynamic structure factor $S(\omega)$. Equation (4) also presents some direct corollaries, such as a sum rule which we discuss in the Supplementary Material.

The identification (4) has several conceptual implications.

First of all, it makes the QFI, a witness for multipartite entanglement, a straightforwardly measurable quantity. Dynamic susceptibilities are routinely measured in manybody systems using well-established techniques such as Bragg spectroscopy [28, 29, or neutron scattering [30.

Second, the central Eq. (4) has also theoretical implications. As one example, the dynamical susceptibility measures quantum fluctuations. Since fluctuations determine the sensitivity of a state towards external perturbations, the existence of a relation to the QFI is intuitive, which now becomes rigorous via Eq. (4). From a different point of view, the QFI extracts the entanglement content of the quantum correlations contained in $\chi^{\prime \prime}(\omega, T)$.

Third, the connection (4) has direct quantitative consequences near continuous QPTs when choosing for $\hat{\mathcal{O}}$ a relevant operator in the renormalisation-group sense, such as the order parameter. Then, known universal scaling laws for $\chi^{\prime \prime}(\omega, T)$ translate directly into universal 
scaling for $F_{Q}$. In magnetic materials, e.g., experiments have observed the scaling $\chi^{\prime \prime}(\omega, T) \sim(T / J)^{-s} \phi(\omega / T)$, with $\phi$ a universal function and $s$ a scaling exponent (see, e.g., 31, 32). Integrating this, one directly obtains the scaling $f_{Q} \sim(T / J)^{1-s}$. Universal scaling has already been observed theoretically for the QFI in ground states of many-body models [17 20 , and has been characterized for the geometric tensor in the ground state [21, 22 . as well as thermal states 23. In the following, we discuss the general scaling behaviour of the QFI in the experimentally relevant regime of non-zero temperatures.

\section{Universal scaling of multipartite entanglement}

Consider a local generator $\hat{\mathcal{O}}=\sum_{l=1}^{N} \hat{\mathcal{O}}_{l}$, in a $d-$ dimensional system with linear size $L$, lattice spacing $a=1$, and $N=L^{d}$ sites. As explained in the Supplementary Material, the universal behaviour of $f_{Q}$, following standard scaling arguments, is

$$
f_{Q}\left(T / J, L^{-1}, \tilde{h}\right)=\lambda^{\Delta_{Q}} \phi_{Q}\left(\lambda^{z} T / J, \lambda L^{-1}, \lambda^{1 / \nu} \tilde{h}\right) .
$$

Here, $z$ is the dynamical and $\nu$ the correlation-length critical exponent. $\lambda$ is the cutoff scale determined by the relevant perturbations $L^{-1} 2 T$, and the normalized distance from the critical point $h$. The scaling dimension of $f_{Q}$ is $\Delta_{Q}=d-2 \Delta_{\alpha}$, with $\Delta_{\alpha}$ the scaling dimension of $\hat{\mathcal{O}}_{l}$.

Since $f_{Q}$ bounds the number of entangled particles via Eq. (3), its scaling behaviour allows us to identify a class of strongly entangled QPTs, i.e., QPTs with a divergent multipartiteness of entanglement. These are those transitions with $\Delta_{Q}>0$. The scaling behaviour additionally implies a length scale $l_{\text {ent }}$ 33] over which multipartite entanglement as measured by the QFI exists, $l_{\text {ent }} \gtrsim f_{Q}^{1 / d} \sim \lambda^{1-2 \Delta_{\alpha} / d}$ (see Supplementary Material).

The framework described up to now is completely general. To illustrate its viability for understanding entanglement in quantum many-body systems, we now turn to specific examples. We focus on a paradigmatic class of model systems exhibiting a QPT, namely Ising models in a transverse field, which are realisable in quantum-optical [10, 16] as well as solid-state systems [14, 15, 34,

$$
\frac{H}{J}=-\cos (\gamma) \sum_{l, j=1}^{N} \mathcal{J}_{l j} \sigma_{l}^{x} \sigma_{j}^{x}+\sin (\gamma) \sum_{l=1}^{N} \sigma_{l}^{z} .
$$

Here, $\sigma_{l}^{\alpha}, \alpha=x, y, z$, is the Pauli matrix on lattice site $l$. Depending on the interactions $\mathcal{J}_{l j}$, this Hamiltonian exhibits a quantum critical point at some critical field strength $\gamma_{c}$. The order parameter for the transition is $\sum_{l}\left\langle\sigma_{l}^{x}\right\rangle / N$. Its strong critical fluctuations make the generator $\hat{\mathcal{O}}=\sum_{l} \sigma_{l}^{x} / 2$ an ideal candidate for testing the scaling behaviour of the QFI.

We first focus on the simplest case, the onedimensional nearest-neighbour Ising chain, $\mathcal{J}_{l j}=\delta_{j, l+1}$, where the scaling exponents are known analytically, $z=$ 1 and $\Delta_{\alpha}=1 / 8$ [34]. This gives $\Delta_{Q}=3 / 4$, i.e., the nearest-neighbour quantum Ising chain lies in the class

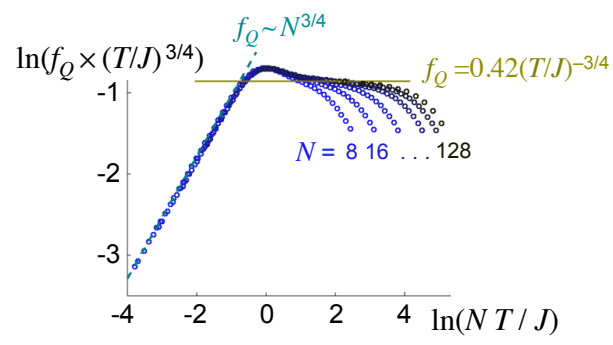

Figure 2. Universal scaling of the quantum Fisher information density, $f_{Q}=F_{Q} / N$, calculated for the order parameter in the quantum Ising chain. Exact data at the critical point and for $N=8 \ldots 128$. At low temperatures, the universal scaling laws produce an excellent data collapse. In a range of intermediate temperatures, the scaling approaches analytic predictions for the thermal critical regime in the thermodynamic limit 34 . (solid line). The strong divergence at small temperatures of $f_{Q} \sim N^{3 / 4}$ (dashed line) implies a diverging multipartiteness of entanglement.

of strongly entangled phase transitions with divergent multipartite entanglement. Indeed, $f_{Q}$ for the order parameter displays a strong peak around the critical point $\gamma_{c}=\pi / 4$, see Fig. 1.(c). The entanglement radiates out from the peak, generating a broad entangled region also at non-zero $T$ [35, 36.

To illustrate the Ansatz (7), we consider the scaling with system size $L$ and temperature $T$, at fixed $\tilde{h}=0$. For $L \ll(T / J)^{-1 / z}$, the dominant cutoff scale is $\lambda \sim$ $L=N$, implying

$$
f_{Q} \sim N^{3 / 4}
$$

The data in Fig. 2 reproduces perfectly this strong algebraic growth, which is remarkably close to the theoretical maximum of $f_{Q}=N$. The associated multipartite entanglement length scale is thus highly divergent, $l_{\text {ent }} \sim N^{3 / 4}$.

With increasing temperature, the cutoff scale crosses over to $\lambda \sim(T / J)^{-1 / z}$, and the scaling becomes $f_{Q}=C(T / J)^{-3 / 4}$. The constant $C \approx 0.42$ can be obtained using analytical results for the dynamic susceptibility at criticality in the thermodynamic limit 34 (see Methods). In the temperature regime of validity, $N T / J \gg 1$ and $T / J \ll 1$, the exact data for finite chains is consistent with this scaling prediction (see Fig. 2). For $T \gg J$ the system crosses over into a generic high-temperature asymptotic behaviour $f_{Q} \sim(T / J)^{-2}$.

\footnotetext{
Absence of signature at thermal phase transitions Remarkably, scaling behaviour is only observed at quantum, but not thermal phase transitions, because Eq. (4) considers only quantum fluctuations. A simple example to demonstrate the insensitivity towards thermal phase transitions is provided by the fully-connected transversefield Ising model, $\mathcal{J}_{l j}=1 / N, \forall l, j$ [17, 37, similar to the model describing the experiments of Ref. [10]. In contrast to its nearest-neighbour counterpart, this model exhibits, additionally to the QPT at $\gamma_{c}=\pi / 4$, also a thermal phase transition 38 (see Methods).
} 

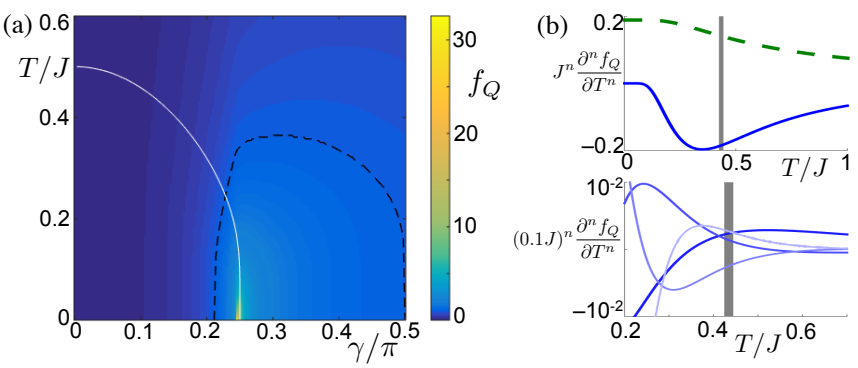

Figure 3. Absence of features of the quantum Fisher information at thermal phase transitions, exemplified by the infinitely-connected Ising model. (a) Finite entanglement is witnessed at non-zero temperatures (enclosed by the dashed line), and a divergence appears near the quantum critical point at $\gamma=\pi / 4$ and $T=0$. But, crucially, $f_{Q}$ shows no features at the thermal phase transition (solid line). Exact data for $N=1000$. (b) Derivatives of $f_{Q}$ remain also smooth at the thermal transition (vertical line). Top: $f_{Q}$ (dashed) and $\partial f_{Q} / \partial(T / J)$ (solid line). Bottom, from dark to light: second to fifth derivative. Exact data for $N=1600$ and $\gamma=\pi / 8$.

Figure 3(a) shows $f_{Q}$ for the order parameter in the temperature-transverse-field plane. We delegate its scaling analysis to the Supplementary Material. More important at this point, while $f_{Q}$ shows a divergence at the QPT, no particular feature can be discerned at the thermal phase transition. Neither do such features appear in derivatives of $f_{Q}$ [Fig. 33(b)].

However, it is known that the static isothermal susceptibility $\chi^{T}$ of the order parameter diverges [39. The reason why the QFI instead remains featureless becomes clear when decomposing $\chi^{T}=\chi_{\mathrm{el}}+\chi_{\mathrm{vV}}$ into its two fundamental parts [40, the elastic (or Curie) contribution $\chi_{\mathrm{el}}$, and the quantum-mechanical van-Vleck correction $\chi_{\mathrm{vV}}$, which is continuously connected to the Kubo susceptibility, $\chi_{\mathrm{vV}}=\lim _{\omega \rightarrow 0} \chi(\omega, T)$. It is $\chi_{\mathrm{el}}$ that diverges at a thermal phase transition. As is shown in the Supplementary Material, $\chi_{\mathrm{el}}$ can be related to the Fisher information in a classical scenario [41] that has no relation to entanglement. The second term $\chi_{\mathrm{vV}}$, on the other hand, remains smooth at thermal transitions. The QFI, thus, considers only the contribution to the susceptibility that is due to quantum fluctuations and remains insensitive to thermal phase transitions.

\section{Experimental considerations}

Let us finally address some practical aspects that will be important for experiments. The measurement prescription (4) is very flexible, since its proof did not make any assumptions on microscopic details of the system under study (other than thermal equilibrium). As a consequence, it applies in any spatial dimension, for any hermitian generator $\hat{\mathcal{O}}$, and it can be equally used for systems of spins, bosons, or fermions. A bound for multipartite spatial entanglement as in Eq. (3) is known to exist in all cases where the generator is a sum of local operators, $\hat{\mathcal{O}}=\sum_{l} \hat{\mathcal{O}}_{l}$, when $\hat{\mathcal{O}}_{l}$ has a bounded spectrum [13] (see
Methods). In this context, it is important to note that the scaling analysis overcomes a usual practical difficulty for studying the QFI, the optimal choice for the linear generator. In the vicinity of a QPT, the choice becomes clear: one may select any suitable, relevant operator $\hat{\mathcal{O}}_{l}$, preferably the one with the largest scaling exponent $\Delta_{Q}$. A good choice will often be the order parameter, as in the examples above. The Supplementary Material contains an example for the Mott-insulator-superfluid transition, where universal behaviour is extracted from an operator different from the order parameter that is accessible by Bragg spectroscopy. Importantly, once an appropriate generator is chosen, the complexity of measuring $\chi^{\prime \prime}(\omega, T)$ does not scale with system size, thus allowing for an efficient evaluation of the QFI via Eq. (4).

Remarkably, the measurement prescription is robust against intrinsic sources of imperfections. For example, an uncertainty in determining the system's temperature can be mitigated by choosing the largest temperature estimate. Due to the monotonicity of the $\tanh (\omega / 2 T)$ as a function of frequency, this produces a lower bound for the QFI. Another natural error source is a finite spectral resolution. Assuming a symmetric broadening function (such as the Gaussians commonly used in neutron spectroscopy [30]), the concavity of the tanh assures that the integration again returns a lower bound to the true QFI, with less severe consequences at smaller temperatures. Also, the integral in Eq. (4) will be limited to some frequency range (the upper integral limit represents, as usual, a scale much larger than energies accessible by the considered degrees of freedom). This limitation will again deliver a lower estimate. Remarkably, none of these errors will produce a false positive indicator for entanglement. The integration in Eq. (4) thus entails a certain intrinsic robustness, as compared to more error-prone measurements at precisely one fixed $\omega$. What is more, the existence of the universal scaling laws assures close to a QPT an additional inherent robustness of the QFI. Therefore, in contrast to some engineered highly-entangled states, such as the GHZ state, the entanglement witnessed by the QFI close to QPTs is unaffected by weak symmetrypreserving perturbations.

The dynamic susceptibility has been studied experimentally in a large variety of strongly-correlated materials (see, e.g., [14, 31, 32, 42, 44]). It will be worthwhile to revisit such experiments in light of Eq. (4). While it is challenging in neutron spectroscopy to measure absolute values, they can be obtained by appropriate normalisation, e.g., via incoherent elastic or phonon scattering [45, allowing neutron scattering with current instrumentation to account for 99(8)\% of the spectral weight [46. Even more, the general scaling behaviour of multipartite entanglement may be already estimated without such precise normalisation, and even from existing data. For example, measurements in certain quantum-critical magnetic materials are consistent with a scaling exponent $\Delta_{Q}>0$ 31, 32, as we require for a divergent QFI. Scaling behaviour for 
dynamic susceptibilities can sometimes even be observed in absence of QPTs, such as in certain heavy-fermion compounds at elevated temperatures [42].

\section{Prospects}

The proposed measurement procedure is scalable to large particle numbers, and is thus complementary to exponentially expensive approaches such as Refs. [6, 7]. While the latter can in principle access non-local entanglement, as characterizes, e.g., topological phases [25, 26, physical susceptibilities are related to local operators. Hence, though the QFI does display scaling behaviour at topological transitions, it does not identify topological phases, as we illustrate in the Supplementary Material using the example of the Kitaev wire. It shares this fate with other entanglement witnesses [1, 47] (for some recent applications to quantum many-body experiments, see Refs. 4851]). A remaining challenge is the question whether nonlocal extensions of the proposed protocol permit the characterization of topological entanglement 26] and exotic quantum phases [25, 43].

The multipartite entanglement that the QFI detects has an immediate interpretation as a resource for quantum metrology [8, 9. Further potential applications range from quantifying the entanglement in quantum simulators of many-body problems $[52,54$ to the characterization of strongly-correlated systems. For example, a natural question is whether entanglement influences such striking material properties as high-temperature superconductivity, where scaling behaviour has already been observed in the optical conductivity [55. Specifically, an underlying antiferromagnetic critical point 44] will show quantum-critical scaling of entanglement, which may also be tested in cold atomic-gas experiments [56, 57.

\section{Author Information}

Reprints and permissions information is available at www.nature.com/reprints. The authors declare no competing financial interests. Readers are welcome to comment on the online version of the paper. Correspondence and requests for materials should be addressed to P.H. (philipp.hauke@uibk.ac.at).

\section{Acknowledgements}

We thank E. Bertel, J.I. Cirac, R. Fazio, M. Mourigal, L. Pezzé, and A. Smerzi for useful discussions. We acknowledge support from the EU IP SIQS, SFB FoQuS (FWF Project No. F4016-N23), the ERC synergy grant UQUAM, the Deutsche Akademie der Naturforscher Leopoldina (grant No. LPDS 2013-07 and LPDR 201501), Spanish Government Grant FOQUS, ERC AdG OSYRIS, EU STREP EQuaM, and EU FET Proactive QUIC. The numerical evaluation of Pfaffians uses the algorithm provided in Ref. [58.

\section{Author Contributions}

All authors devised the project, discussed the results, and wrote the manuscript.

\section{Methods}

\section{Relation of QFI to multipartite entanglement}

The QFI has a deep connection with multipartite entanglement [8, 9. Consider a system of $N$ spins with length $S=\frac{1}{2}$, and a linear observable $\hat{\mathcal{O}}=\frac{1}{2} \sum_{l} \mathbf{n}_{l} \cdot \boldsymbol{\sigma}_{l}$, where $\boldsymbol{\sigma}_{l}=\left(\sigma_{l}^{x}, \sigma_{l}^{y}, \sigma_{l}^{z}\right)$ is a vector containing the Pauli matrices $\sigma_{l}^{\alpha}$ associated to spin $l$, and $\mathbf{n}_{l}=\left(n_{l}^{x}, n_{l}^{y}, n_{l}^{z}\right)$ is a unit vector on the Bloch sphere. Then the system hosts at least $m+1$-partite entanglement if the QFI associated to $\hat{\mathcal{O}}$ fulfils 8

$$
F_{Q}>\left\lfloor\frac{N}{m}\right\rfloor m^{2}+\left(N-\left\lfloor\frac{N}{m}\right\rfloor m\right)^{2},
$$

where $\lfloor X\rfloor$ is the largest integer smaller than or equal to $X$. For $m$ a divisor of $N$, the condition (10) attains the simple form (3) when expressed through the QFI density $f_{Q} \equiv F_{Q} / N$. Note that typically response functions that measure linear operators (at fixed wave-vector transfer $q$ ) contain a factor of $1 / N$ relative to our definition, Eq. (5), so they will observe directly $f_{Q}$ rather than $F_{Q}$.

The proof of Ref. 8 for bounding the multipartite entanglement with Eq. (10) can be directly translated to degrees of freedom other than spins $1 / 2$, as long as $\hat{\mathcal{O}}$ represents a sum of local operators with bounded spectrum [13]. If $h_{\max }$ and $h_{\min }$ denote the largest, respectively smallest, eigenvalue of $\hat{\mathcal{O}}$, then the right hand side of the condition (10) acquires the prefactor $\left(h_{\max }-h_{\min }\right)^{2}$. Therefore, the QFI can also witness spatial entanglement in systems other than spins-1/2, such as larger spins or fermions. Note that the relation of the QFI to response functions, Eq. (4), is independent of any such microscopic details of the underlying quantum many-body system or the hermitian operator $\hat{\mathcal{O}}$, which may even be non-local or unbounded.

\section{Solvability of the considered models}

All models used in this article for illustrating the main concepts are exactly solvable (throughout we set $\hbar=$ $k_{B}=a=1$, with $a$ the lattice spacing). The onedimensional Ising chain in a transverse field [Eq. (8) with $\left.\mathcal{J}_{l j}=\delta_{j, l+1}\right]$ can be mapped to a free-fermion problem [34. Dynamical susceptibilities for finite systems can then be calculated via Wick's decomposition of expectation values 59, and the Pfaffians appearing in the resulting expressions can be evaluated efficiently using the algorithm described in Ref. [58]. Exact results for finite systems with the order parameter as generator are displayed in Figs. 11(c) and 2

Moreover, for the transverse Ising chain in the thermodynamic limit, analytical results exist for the orderparameter dynamic susceptibility in the quantum-critical region 34. For wavevector transfer $k=0$, at non-zero 
temperatures above the critical point $(\tilde{h}=0)$ the dynamic susceptibility reads

$$
\lim _{N \rightarrow \infty} \frac{\chi(\omega)}{N}=c \frac{J^{3 / 4}}{T^{7 / 4}}\left(\frac{\Gamma\left(\frac{1}{16}-i \frac{\omega}{4 \pi T}\right)}{\Gamma\left(\frac{15}{16}-i \frac{\omega}{4 \pi T}\right)}\right)^{2},
$$

where $c \approx 3.81 \cdot 10^{-3}$ and $\Gamma$ denotes the Gamma-function. Performing the $\omega$-integral in Eq. (4), one immediately obtains the QFI,

$$
f_{Q}(T)=C(J / T)^{3 / 4},
$$

with $C \approx 0.42$. Data based on these equations is presented in Fig. 1.(b) and as one limiting case in Fig. 2,

The infinite-range Ising Hamiltonian [Eq. (8) with $\left.\mathcal{J}_{l j}=1 / N\right]$ commutes with both $\mathbf{S}^{2}=\left(S^{x}\right)^{2}+\left(S^{y}\right)^{2}+$
$\left(S^{z}\right)^{2}$ and $S^{z}$ where $S^{\alpha}=\sum_{l} \sigma_{l}^{\alpha} / 2, \alpha=x, y, z$. As a consequence, the Hamiltonian decomposes into disconnected blocks when represented in the common eigenbasis of $\mathbf{S}^{2}$ and $S^{z}$. Each block grows linearly with particle number $N$ and can be diagonalised efficiently, allowing one to compute the dynamical susceptibility exactly even for large systems. Here, we consider the largest of these blocks, with dimension $N+1$. Figure 3 presents corresponding exact data. The infinite-range Ising model has a thermal phase transition for $\gamma<\pi / 4$, with critical temperature 38.

$$
\frac{T_{c}}{J}=\frac{\sin (\gamma)}{\log [(1+\tan (\gamma)) /(1-\tan (\gamma))]}
$$

[1] L. Amico, R. Fazio, A. Osterloh, and V. Vedral, Entanglement in many-body systems. Rev. Mod. Phys. 80, 517-576 (2008).

[2] K. Kim, M.-S. Chang, S. Korenblit, R. Islam, E. E. Edwards, J. K. Freericks, G.-D. Lin, L.-M. Duan, and C. Monroe, Quantum simulation of frustrated Ising spins with trapped ions. Nature 465, 590-593 (2010).

[3] P. Jurcevic, B. P. Lanyon, P. Hauke, C. Hempel, P. Zoller, R. Blatt, and C. F. Roos, Quasiparticle engineering and entanglement propagation in a quantum many-body system. Nature 511, 202-205 (2014).

[4] T. Lanting et al., Entanglement in a quantum annealing processor. Phys. Rev. X 4, 021041 (2014).

[5] T. Fukuhara, S. Hild, J. Zeiher, P. Schauß, I. Bloch, M. Endres, and C. Gross, Spatially Resolved Detection of a Spin-Entanglement Wave in a Bose-Hubbard Chain. Phys. Rev. Lett. 115, 035302 (2015).

[6] A. J. Daley, H. Pichler, J. Schachenmayer, and P. Zoller, Measuring entanglement growth in quench dynamics of bosons in an optical lattice. Phys. Rev. Lett. 109, 020505 (2012).

[7] R. Islam, R. Ma, P. M. Preiss, M. E. Tai, A. Lukin, M. Rispoli, and M. Greiner, Measuring entanglement entropy through the interference of quantum many-body twins. arXiv:1509.01160 [cond-mat.quant-gas] (2015).

[8] P. Hyllus, W. Laskowski, R. Krischek, C. Schwemmer, W. Wieczorek, H. Weinfurter, L. Pezzé, and A. Smerzi, Fisher information and multiparticle entanglement. Phys. Rev. A 85, 022321 (2012).

[9] G. Tóth, Multipartite entanglement and high precision metrology. Phys. Rev. A 85, 022322 (2012).

[10] H. Strobel, W. Muessel, D. Linnemann, T. Zibold, D. B. Hume, L. Pezzé, A. Smerzi, and M. K. Oberthaler, Fisher information and entanglement of non-Gaussian spin states. Science 345, 424-427 (2014).

[11] S. L. Braunstein and C. M. Caves, Statistical Distance and the Geometry of Quantum States. Phys. Rev. Lett. 72, 3439-3443 (1994).

[12] B. M. Escher, R. L. de Matos Filho, and L. Davidovich, General framework for estimating the ultimate precision limit in noisy quantum-enhanced metrology. Nature Phys. 7, 406-411 (2011).

[13] L. Pezzé and A. Smerzi, Quantum theory of phase es- timation. In "Atom Interferometry", Proceedings of the International School of Physics "Enrico Fermi", Course 188, Varenna, edited by G. Tino and M. Kasevich (IOS Press, Amsterdam, 2014) p. 691-741, arXiv:1411.5164 [quant-ph].

[14] R. Coldea, D. A. Tennant, E. M. Wheeler, E. Wawrzynska, D. Prabhakaran, M. Telling, K. Habicht, P. Smeibidl, and K. Kiefer, Quantum Criticality in an Ising Chain: Experimental Evidence for Emergent E 8 Symmetry. Science 327, 177-180 (2010).

[15] M. Hälg, D. Hüvonen, T. Guidi, D. L. Quintero-Castro, M. Boehm, L. P. Regnault, M. Hagiwara, and A. Zheludev, Finite-temperature scaling of spin correlations in an experimental realization of the one-dimensional Ising quantum critical point. Phys. Rev. B 92, 014412 (2015).

[16] J. Simon, W. S. Bakr, R. Ma, M. E. Tai, P. M. Preiss, and M. Greiner, Quantum Simulation of Antiferromagnetic Spin Chains in an Optical Lattice. Nature 472, 307312 (2011).

[17] J. Ma and X. Wang, Fisher information and spin squeezing in the Lipkin-Meshkov-Glick model. Phys. Rev. A 80, 012318 (2009).

[18] W.-F. Liu, J. Ma, and X. Wang, Quantum Fisher information and spin squeezing in the ground state of the XY model. J. Phys. A: Math. Theor. 46, 045302 (2013).

[19] T.-L. Wang, L.-N. Wu, W. Yang, G.-R. Jin, N. Lambert, and F. Nori, Quantum Fisher information as a signature of the superradiant quantum phase transition. New J. Phys. 16, 063039 (2014).

[20] Q. Zheng, Y. Yao, and X.-W. Xu, Renormalized quantum Fisher information manifestation of BerezinskiiKosterlitz-Thouless phase transition for spin-1/2 XXZ chain. Commun. Theor. Phys. 63, 279-284 (2015).

[21] L. Campos Venuti and P. Zanardi, Quantum Critical Scaling of the Geometric Tensors. Phys. Rev. Lett. 99, 095701 (2007).

[22] W. L. You, Y. W. Li, and S. J. Gu, Fidelity, dynamic structure factor, and susceptibility in critical phenomena. Phys. Rev. E 76, 022101 (2007).

[23] P. Zanardi, L. Campos Venuti, and P. Giorda, Bures metric over thermal state manifolds and quantum criticality. Phys. Rev. A 76, 062318 (2007).

[24] J. Smith, A. Lee, P. Richerme, B. Neyenhuis, P. W. Hess, 
P. Hauke, M. Heyl, D. Huse, and C. Monroe, Many-body localization in a quantum simulator with programmable random disorder. arXiv:1508.07026 [quant-ph] (2015).

[25] L. Balents, Spin liquids in frustrated magnets. Nature 464, 199-208 (2010).

[26] B. Zeng, X. Chen, D.-L. Zhou, X.-G. Wen, Quantum Information Meets Quantum Matter - From Quantum Entanglement to Topological Phase in Many-Body Systems. arXiv:1508.02595 [cond-mat.str-el] (2015).

[27] L. Wang, Y.-H. Liu, J. Imriška, P. N. Ma, and M. Troyer, Fidelity Susceptibility Made Simple: A Unified Quantum Monte Carlo Approach. Phys. Rev. X 5, 031007 (2015).

[28] T. Stöferle, H. Moritz, C. Schori, M. Köhl, and T. Esslinger, Transition from a Strongly Interacting 1D Superfluid to a Mott Insulator. Phys. Rev. Lett. 92, 130403 (2004).

[29] P. T. Ernst, S. Götze, J. S. Krauser, K. Pyka, D.-S. Lühmann, D. Pfannkuche, and K. Sengstock, Probing superfluids in optical lattices by momentum-resolved Bragg spectroscopy. Nature Phys. 6, 56-61 (2010).

[30] G. Shirane, S. M. Shapiro, and J. M. Tranquada, Neutron Scattering with a Triple-Axis Spectrometer, Basic Techniques (Cambridge University Press, 2002).

[31] B. Lake, A. M. Tsvelik, S. Notbohm, D. A. Tennant, T. G. Perring, M. Reehuis, C. Sekar, G. Krabbes, and B. Büchner, Confinement of fractional quantum number particles in a condensed-matter system. Nature Phys. 6, 50-55 (2010).

[32] M. Hälg, D. Hüvonen, N. P. Butch, F. Demmel, and A. Zheludev, Finite-temperature scaling of spin correlations in a partially magnetized Heisenberg $\mathrm{S}=1 / 2$ chain. Phys. Rev. B 92, 104416 (2015).

[33] F. Verstraete, M. Popp, and J. I. Cirac, Entanglement versus Correlations in Spin Systems. Phys. Rev. Lett. 92, 027901 (2004).

[34] S. Sachdev, Quantum Phase Transitions (Cambridge University Press, 1999).

[35] G. Tóth, Entanglement witnesses in spin models. Phys. Rev. A 71, 010301(R) (2005).

[36] L.-A. Wu, S. Bandyopadhyay, M. S. Sarandy, and D. A. Lidar, Entanglement observables and witnesses for interacting quantum spin systems. Phys. Rev. A 72, 032309 (2005).

[37] S. Dusuel and J. Vidal, Finite-Size Scaling Exponents of the Lipkin-Meshkov-Glick Model. Phys. Rev. Lett. 93, 237204 (2004).

[38] A. Das, K. Sengupta, D. Sen, and B. K. Chakrabarti, Infinite-range Ising ferromagnet in a time-dependent transverse magnetic field: Quench and ac dynamics near the quantum critical point. Phys. Rev. B 74, 144423 (2006).

[39] J. Cardy, Scaling and Renormalization in Statistical Physics (Cambridge Lecture Notes in Physics, 1996).

[40] J. Jensen and A. R. Mackintosh, "Rare earth magnetism: Structures and excitations," (Clarendon Press, Oxford, 1991) Chap. Linear Response Theory.

[41] M. Prokopenko, J. T. Lizier, O. Obst, and X. R. Wang, Relating Fisher information to order parameters. Phys. Rev. E 84, 041116 (2011).

[42] W. Knafo, S. Raymond, J. Flouquet, B. Fåk, M. A.
Adams, P. Haen, F. Lapierre, S. Yates, and P. Lejay, Anomalous scaling behavior of the dynamical spin susceptibility of Ce0.925La0.075Ru2Si2. Phys. Rev. B 70, 174401 (2004).

[43] T.-H. Han, J. S. Helton, S. Chu, D. G. Nocera, J. A. Rodriguez-Rivera, C. Broholm, and Y. S. Lee, Fractionalized excitations in the spin-liquid state of a kagomelattice antiferromagnet. Nature 492, 406-410 (2012).

[44] P. Dai, Antiferromagnetic order and spin dynamics in iron-based superconductors. Rev. Mod. Phys. 87, 855-896 (2015).

[45] G. Xu, Z. Xu, and J. M. Tranquada, Absolute crosssection normalization of magnetic neutron scattering data. Rev. Sci. Instrum. 84, 083906 (2013).

[46] M. Mourigal, M. Enderle, A. Klöpperpieper, J.-S. Caux, A. Stunault, and H. M. Rønnow, Fractional spinon excitations in the quantum Heisenberg antiferromagnetic chain. Nature Phys. 9, 435-441 (2013).

[47] O. Gühne and G. Tóth, Entanglement detection. Physics Reports 474, 1-75 (2009).

[48] S. Ghosh, T. F. Rosenbaum, G. Aeppli, and S. N. Coppersmith, Entangled quantum state of magnetic dipoles. Nature 425, 48-51 (2003).

[49] Č. Brukner, V. Vedral, and A. Zeilinger, Crucial role of quantum entanglement in bulk properties of solids. Phys. Rev. A 73, 012110 (2006).

[50] T. Vértesi and E. Bene, Thermal entanglement in the nanotubular system Na2V3O7. Phys. Rev. B 73, 134404 (2006).

[51] M. Cramer, A. Bernard, N. Fabbri, L. Fallani, C. Fort, S. Rosi, F. Caruso, M. Inguscio, and M. Plenio, Spatial entanglement of bosons in optical lattices. Nat. Commun. 4, 2161 (2013).

[52] J. I. Cirac and P. Zoller, Goals and opportunities in quantum simulation. Nature Phys. 8, 264-266 (2012).

[53] P. Hauke, F. M. Cucchietti, L. Tagliacozzo, I. Deutsch, and M. Lewenstein, Can One Trust Quantum Simulators? Rep. Prog. Phys. 75, 082401 (2012).

[54] I. M. Georgescu, S. Ashhab, and F. Nori, Quantum Simulation. Rev. Mod. Phys. 86, 153-185 (2014).

[55] D. van der Marel, H. J. A. Molegraaf, J. Zaanen, Z. Nussinov, F. Carbone, A. Damascelli, H. Eisaki, M. Greven, P. H. Kes, and M. Li, Quantum critical behaviour in a high-Tc superconductor. Nature 425, 271-274 (2003).

[56] D. Greif, T. Uehlinger, G. Jotzu, L. Tarruell, and T. Esslinger, Short-Range Quantum Magnetism of Ultracold Fermions in an Optical Lattice. Science 340, 13071310 (2013).

[57] R. A. Hart, P. M. Duarte, T.-L. Yang, X. Liu, T. Paiva, E. Khatami, R. T. Scalettar, N. Trivedi, D. A. Huse, and R. G. Hulet, Observation of antiferromagnetic correlations in the Hubbard model with ultracold atoms. Nature 519, 211-214 (2015).

[58] M. Wimmer, Efficient numerical computation of the Pfaffian for dense and banded skew-symmetric matrices. ACM Trans. Math. Software 38, 30 (2012).

[59] O. Derzhko and T. Krokhmalskii, Dynamic structure factor of the spin-1/2 transverse Ising chain. Phys. Rev. B 56, 11659-11665 (1997). 